\title{
Chromint Software
}

National Cancer Institute

\section{Source}

National Cancer Institute. Chromint Software. NCI Thesaurus. Code C70843.

A method for detection of chromatography peaks based on the analysis of the profile of the first derivative of the signal calculated with a Savitzky-Golay filter. This provides a list of peaks and their position with typically around 25 peaks detected per electropherogram. 